\title{
Exploration of micro- and macronutrient consumption and leverage of interaction with adipokines among Jordanian adults
}

Background and Aim: Findings related to nutrient intakes and levels of adipokines concentrations are inconclusive. The present study aimed at investigating the association between intakes of macro- and micro-nutrients with serum adipokines in apparently healthy adults.

\begin{abstract}
Methods and Results: A convenient sample of 167 adults was obtained from students and employees in major hospital in Jordan. Serum concentrations of leptin, adiponectin, resistin and interleukine- 6 were measured. Nutrients intakes were assessed using a validated quantitative food frequency questionnaire. Higher levels of leptin were associated with the highest consumption of energy from carbohydrate, insoluble, and soluble fiber $(P=0.04)$. Lower levels of leptin were associated with highest consumption of energy from fat $(\mathrm{P}=0.04)$, monounsaturated fatty acids $(\mathrm{P}=0.04)$ and cholesterol $(P=0.02)$. Lower levels of adiponectin were found among individuals with the highest consumption of carbohydrates $(p=0.02)$ insoluble fibers $(P=0.01)$; and copper $(P=0.03)$. Higher levels of adiponectin were associated with higher consumption of cholesterol $(P=0.03)$. Leptin/adiponectin ratio was positively associated with the intakes of carbohydrates $(P=0.04)$, soluble- $(P=0.01)$ and insoluble fibers $(P=0.01)$ and copper $(P=0.03)$, whereas the ratio was negatively associated with cholesterol $(P=0.04)$, butyric acid $(P=0.03)$ and omega-3 fatty acids $(P=0.03)$. Levels of resistin were only associated with total fiber intake $(P=0.04)$ and levels of interleukine- 6 were only associated with cholesterol intake $(P=0.01)$.
\end{abstract}

Conclusion: Our findings suggest that intakes of carbohydrates, fat, cholesterol and fibers are the major dietary factors that may be associated with levels of leptin and adiponectin. Levels of resistin and interleukine- 6 may be less associated with diet composition.

Keywords: macronutrients, micronutrients, leptin, resistin, IL-6, adiponectin, leptin/adiponectin ratio

\section{Introduction}

Adipose tissue is the major site for storage of excess energy in the form of triglycerides, and it contains different cell types, including mainly adipocytes, preadipocytes, endothelial cells and immune cells [1]. Adipose tissue has been recognized as an active endocrine organ secreting a variety of adipokines, which are bioactive peptides that can induce several autocrine/paracrine or endocrine effects [2]. Adipokines include cytokines e.g., tumor necrosis factor (TNF)- $\alpha$, interleukins (IL-1, IL-6 and IL-10), lipid-related peptides, several enzymes, low molecular weight proteins such as leptin, adiponectin, resistin and visfatin [2-4]. Adipokines are responsible for regulating several physiological processes and functions, including food intake balance and energy homeostasis [3], insulin sensitivity [5,6], cardio and vascular protection $[7,8]$. For example, the protein leptin is a satiety adipokine that controls body weight by regulating appetite and energy expenditures [9-11]. Adiponectin has positive association with insulin sensitivity [12], whereas, resistin, visfatin and IL-6 have been identified as proinflammatory mediators, and are linked to insulin resistance and type 2 diabetes [13-15]. Obesity and adipocyte dysfunction result in a disruption of adipokine production, which may contribute to the development of obesitylinked metabolic and cardiovascular diseases via altered glucose and lipid homeostasis as well as inflammatory responses $[16,17]$.

Several studies demonstrated a strong association between dietary intakes of several nutrients with circulating adipokine concentrations in the general population $[18-$ 20]. Some of these nutrients includeomega-3 fatty acid [21,22], protein [23], sucrose [24], and dietary $[22,25]$, or foods such as vegetables and fruits [26], whole grain [27], fish [28,29], and legumes [30]. However, other studies showed no association between some dietary component $[\mathrm{s}]$ such as vitamins and minerals with adipokine levels [31-33]. Studies examined the effect of dietary intake of vitamins and minerals on serum

\author{
Reema F Tayyem ${ }^{* 1}$, \\ Nawal S Hijjawi², \\ Hiba A Bawadi ${ }^{3}$, \\ Nisreen A Nimer ${ }^{4}$, \\ Lana M Agraib ${ }^{1}$, \\ Sabika S Allehdan ${ }^{1}$, \\ Shatha Hammad ${ }^{5}$ \& \\ Ali M Al-Radaideh ${ }^{6}$ \\ ${ }^{1}$ Department of Nutrition and Food \\ Technology, Faculty of Agriculture, The \\ University of Jordan, Amman, Jordan \\ ${ }^{2}$ Department of Medical Laboratory \\ Sciences, Faculty of Allied Health \\ Sciences, The Hashemite University, \\ Zarqa, Jordan \\ ${ }^{3}$ Department of Human Nutrition, \\ College of Health Sciences, Qatar \\ University, Doha, Qatar \\ ${ }^{4}$ Department of Chemistry, College \\ of Sciences and Health Professions, \\ Cleveland State University, Cleveland, \\ Ohio, USA \\ ${ }^{5}$ Department of Human Nutritional \\ Sciences, University of Manitoba, \\ Winnipeg, Manitoba, Canada \\ ${ }^{6}$ Department of Medical Imaging, \\ Faculty of Allied Health Sciences, The \\ Hashemite University, Zarqa, Jordan \\ *Author for correspondence: \\ r.tayyem@ju.edu.jo
}


adipokines are scarce. Al-Daghri et al. (2012) found that increasing levels of dietary vitamin B6 and $\mathrm{B} 12$ have been associated with a significant increase in serum concentrations of leptin but not resistin among Saudi adults and children [34]. Levels of IL-6 and leptin were inversely associated with participants' usual dietary zinc intake and $30 \mathrm{mg} /$ day of zinc supplementation significantly decreased IL-6 level, whereas serum leptin and plasma adiponectin concentration did not differ with either zinc supplementation or placebo among obese women [35]. Recent observational studies showed favorable effects of the Mediterranean dietary pattern, a lowsaturated fat and a high-monounsaturated fat diet, on serum adiponectin, leptin and resistin among US and Spain populations [36-38]. In contrast, high-fructose diets were associated with adverse outcomes such as low adiponectin levels and high leptin levels [39,40].

Jordan is a middle-income country located in a region of the world where dietary habits changed to western pattern considerably. Accordingly, differences and changes in dietary habits and traditional foods between populations may influence the level of those adipokines widely. To the best of our knowledge, no studies assessed the association between nutrients' intake and the following serum adipokines: leptin, resistin, IL-6, adiponectin and leptin/ adiponectin ratio among healthy adults. Therefore, the present study aimed at examining the relationship between macronutrients and micronutrients intakes and serum adipokines levels in apparently healthy Jordanian adults.

\section{Methods \\ - Study design and participants}

In this cross-sectional study, a total number of 167 (83 males and 84 females) apparently healthy Jordanian volunteers (students of Hashemite University and employees of the King Hussein Medical Center (KHMC)), aged 18-51 years were recruited conveniently during the period of October 2014 to July 2015. Sample size was calculated based on an alpha probability of 0.05 and power of 0.8 . Eligibility criteria to be enrolled in the study were: being Jordanian and above 18 years old. Pregnant and lactating women and individuals with eating disorders, major surgeries or any chronic diseases were excluded from this study. A signed informed consent was obtained from each participant. The study protocol was approved by the Jordanian Royal Medical Services (JRMS) ethics committee.

\section{- Measurement of adipokines}

Blood samples were drawn from participants after overnight fasting by a specialized medical laboratory technician. Serum samples were centrifuged and separated from the whole blood and stored at $-80^{\circ} \mathrm{C}$ until further analysis. Serum inflammatory cytokine interleukine-6 (IL-6), adiponectin, resistin and leptin concentrations were measured By ELX 800 TC models 96-well Elisa Microplate Readers-USA using commercially available enzyme-linked immunosorbent assay (ELISA) kits (RayBio ${ }^{\circ}$ Human IL-6 ELISA Kit, USA, Cat\# ELHIL-6; RayBio ${ }^{\circ}$ Human Acrp30 ELISA Kit USA, Cat\# ELH-Adiponectin; RayBio ${ }^{\circ}$ Human Leptin ELISA Kit, USA, Cat\# ELH-Leptin; RayBio $^{\bullet}$ Human Resistin ELISA Kit, USA, Cat\# ELH-Resistin). The reproducibility of the intra- and inter-assay coefficients of variation were $<10 \%$ and $<12 \%$, respectively, for all RayBio $^{\circ}$ ELISA kits. Leptin/adiponectin ratio was calculated as leptin concentration level and divided by adiponectin concentration/level after exchanging of units.

\section{Assessment of nutrients' intake}

A validated Arabic quantitative FFQ adapted from the Diet History Questionnaire I (DHQ I) was used for dietary assessment [41]. This FFQ was used as an assessment tool to estimate nutrients consumption among our study subjects. The FFQ questions sought to obtain information on the dietary history of participants during the 12 months prior to the commencement of the study. We estimated that dietary choices during the last 12 months would be indicative of a fixed habitual pattern reflecting prior years. Participants were asked how frequently, on average, during the past year they had consumed one standard serving of specific food items in nine categories $(<1 /$ month, 2-3/month, 1-2/week, 3-4/week, 5-6/ week, 1/day, 2-3/day, 4-5/day, or 6/day). Food lists in the modified FFQ questions were classified based on types of food: 21 items of fruits and juices; 21 items of vegetables; eight items of cereals; nine items of milk and dairy products; four items of beans; 16 items of meat such as red meat (lamb and beef), chicken, fish, cold meat, and others; four items of soups and sauces; five items of drinks; nine items of snacks and sweets; and 14 items of herbs and spices [41]. For better portion size estimation, food models and standard measuring tools were used. 
Dietary intakes were analyzed using dietary analysis software (ESHA Food Processor SQL version 10.1.1; ESHA, Salem, OR, USA) with additional data on foods consumed in Jordan [42]. For calculating energy and nutrients intake from the food frequency questionnaire, the frequencies of food consumed were transferred into grams. Then those grams from each food item entered to the food processor program ESHA for each person participating in the study. The average for each nutrient consumed by the all participants was then calculated.

\section{- Statistical analysis}

The data were analyzed using SPSS statistical package version 20. Energy, macronutrients and micronutrients were presented as mean \pm standard error. Leptin, resistin, IL-6, adiponectin, leptin/adiponectin ratio were grouped into tertiles. Tertiles were generated using the SPSS statistical program in which the program defines tertile as 33 and 66 percentiles of the nutrients consumed. Therefore, T1 represents less than 33, T2 between 33-66, and T3 above 66 percentiles of the amount of nutrients consumed by the study participants. Post-hoc Analysis of Variance (ANOVA) was used to assess the impact of energy, macronutrients and micronutrients intake on serum adipokines after adjustment for age, sex, BMI, energy intake, physical activity and smoking. Different letters denotes significant differences among the tertiles. P-values $<0.05$ were considered statistically significant.

\section{Results}

Participants' demographic and anthropometric data, the concentration / level of leptin, resistin, IL-6, and adiponectin were published previously in another publication [43]. The authors reported significant statistical differences $(\mathrm{P}<0.01)$ between normal body weight, overweight and obese participants in serum leptin, resistin, IL-6 and adiponectin. The serum levels of leptin, resistin and IL-6 were significantly $(\mathrm{P}<0.01)$ greater in obese participants than those levels reported for overweight and normal body weight participants. Adiponectin serum concentrations were significantly lower in obese participants compared to overweight and normal body weight participants.

The association between participants' daily total energy intake and macronutrients' contribution to energy intake in relation to their serum adipokines and IL- 6 is presented in
TABLE 1. Leptin was significantly associated with the highest consumption of energy from carbohydrate among participants in the highest tertile $(\mathrm{P}=0.04)$. On the contrary, participants in the highest tertiles of adiponectin had the lowest consumption of energy from carbohydrate $(\mathrm{P}=0.03)$. Leptin was significantly associated with the highest energy intake from fat among participants in the lowest and middle tertiles $(\mathrm{P}=0.04)$. There was no significant association between total energy and protein consumption across different tertiles of leptin, resistin, IL-6, adiponectin and leptin/adiponectin ratio. Furthermore, there was no statistically significant relationship with total energy and macronutrient distribution consumptions across all tertiles for resistin and IL-6.

TABLE 2 shows participants' daily intake of different macronutrients through different tertiles of serum adipokines and IL-6. Higher levels of leptin were significantly associated with the higher intake of insoluble fiber $(\mathrm{P}=0.01)$, where lower levels of leptin were associated with the higher intake of monounsaturated fatty acids $(\mathrm{P}=0.04)$ and cholesterol $(\mathrm{P}=0.02)$. Only one significant difference was observed between the daily intakes of different macronutrients across all tertiles of resistin and IL-6. Participants in the lowest and highest tertiles of resistin had the highest intake of fiber compared to participants in the middle tertile $(\mathrm{P}=0.04)$. Lower levels of IL-6 were significantly associated with an increased consumption of cholesterol compared to higher level $(\mathrm{P}=0.01)$. Adiponectin was positively associated with cholesterol $(\mathrm{P}=0.03)$ and negatively with total carbohydrate $(\mathrm{P}=0.02)$ and insoluble fiber $(\mathrm{P}=0.01)$. The consumption of total carbohydrate $(\mathrm{P}=0.04)$, soluble fiber $(\mathrm{P}=0.01)$, and insoluble fiber $(\mathrm{P}=0.01)$ were positively associated with leptin/adiponectin ratio. Conversely, the leptin/adiponectin ratio was negatively associated with cholesterol $(\mathrm{P}=0.04)$, butyric fatty acid $(\mathrm{P}=0.03)$ and omega-3 fatty acids $(\mathrm{P}=0.04)$. Participants in the highest tertile of the adiponectin had significantly the lowest intake of copper when compared to participants in the lowest tertile $(\mathrm{P}=0.03)$. The higher leptin/adiponectin ratio was associated with an increased level of copper consumption compared to the lower ratio, where the difference reached a conventional level for statistical significance $(\mathrm{P}=0.03)$. There was no statistically significant association with other fat soluble and water soluble vitamins as well as calcium, iron and zinc intake through all tertiles of the analyzed serum adipokines (TABLE 3). 


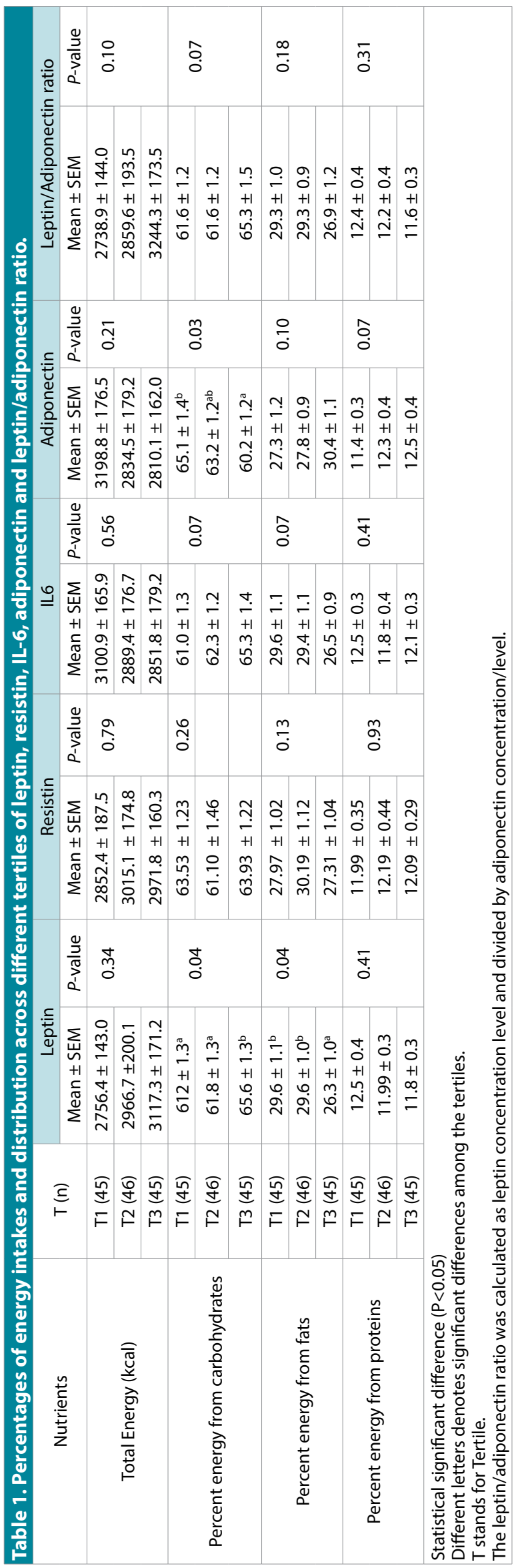




\begin{tabular}{|c|c|c|c|c|c|c|c|c|c|c|c|c|c|c|c|c|c|c|c|c|}
\hline 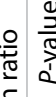 & 童 & & & $\begin{array}{l}0 \\
\stackrel{\infty}{0} \\
0\end{array}$ & & $\stackrel{\overbrace{}}{\circ}$ & & $\bar{o}$ & & & : & & ¿্口: & & ڤn & & $\stackrel{\text { no }}{0}$ & & 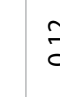 & \\
\hline 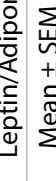 & 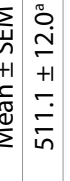 & 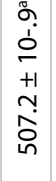 & 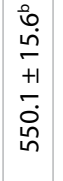 & 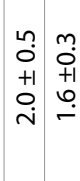 & $\begin{array}{l}n \\
0 \\
+1 \\
o \\
-\end{array}$ & 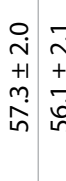 & 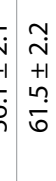 & $\begin{array}{l}0 \\
0 \\
0 \\
+1 \\
\hat{0}\end{array}$ & $\begin{array}{l}\stackrel{o}{0}_{0} \\
+1 \\
+1 \\
\hat{0}\end{array}$ & $\begin{array}{l}0.0 \\
0 \\
0 \\
+1 \\
o \\
\infty \\
\infty\end{array}$ & 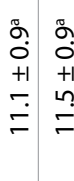 & 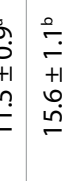 & 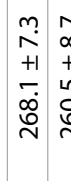 & 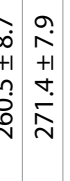 & 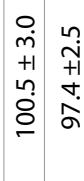 & 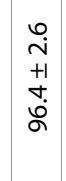 & \begin{tabular}{l|l}
$\stackrel{0}{+}$ & \\
+1 & \\
$\stackrel{1}{n}$ & $\vdots$ \\
$\stackrel{n}{=}$ & $\vdots$
\end{tabular} & & & $\begin{array}{l}\tilde{N} \\
+1 \\
a \\
\\
n\end{array}$ \\
\hline & 童 & & & $\begin{array}{l}\stackrel{L}{\infty} \\
0 \\
0\end{array}$ & & $\stackrel{\infty}{\circ}$ & & $\stackrel{\infty}{0}$ & & & $\overline{0}$ & & ò & & $\frac{5}{O}$ & & $\stackrel{0}{\circ}$ & & $\stackrel{\square}{0}$ & \\
\hline & 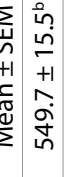 & 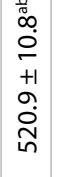 & 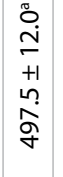 & \begin{tabular}{lll}
$n$ & \multicolumn{1}{c}{} \\
0 & 0 \\
+1 & 0 \\
+1 & +1 \\
\hdashline & 0 \\
- & $\stackrel{-}{-}$ \\
&
\end{tabular} & $\begin{array}{l}n \\
0 \\
+1 \\
+1 \\
o \\
-\end{array}$ & $\begin{array}{cc} & 0 \\
+ & i \\
+1 & +1 \\
m & i \\
i & i \\
& i n\end{array}$ & 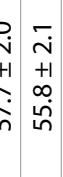 & $\begin{array}{l}\hat{0} \\
+1 \\
+1 \\
\omega \\
\infty\end{array}$ & $\begin{array}{l}0 \\
\stackrel{0}{+} \\
+1 \\
m \\
\end{array}$ & $\begin{array}{l}\tilde{U} \\
0 \\
+1 \\
0 \\
n \\
0\end{array}$ & 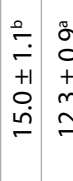 & 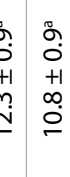 & 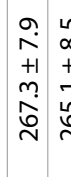 & 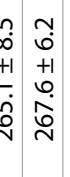 & 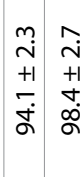 & $\begin{array}{l}0 \\
\stackrel{m}{1} \\
+1 \\
\stackrel{1}{0} \\
- \\
-\end{array}$ & 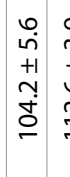 & 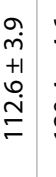 & 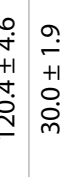 & $\begin{array}{l}n \\
\stackrel{n}{2} \\
+1 \\
\stackrel{a}{\mathbf{n}} \\
m\end{array}$ \\
\hline & & & & ঃ̊ & & 惫 & & $\overline{0}$ & & & ָָ̀ & & 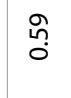 & & $\stackrel{\frac{n}{0}}{\circ}$ & & ָ̃ & & 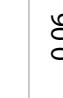 & : \\
\hline
\end{tabular}

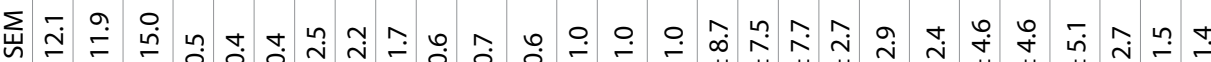

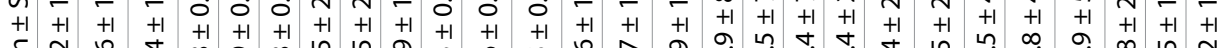

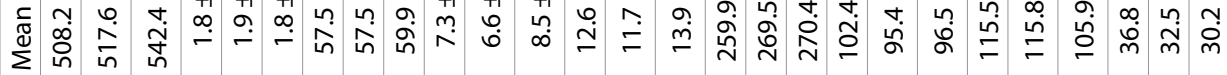

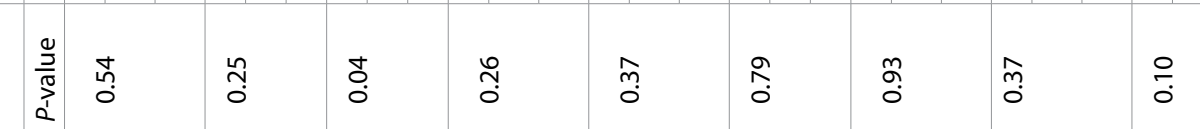

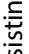

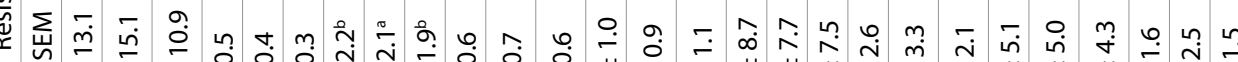

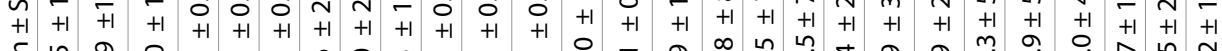

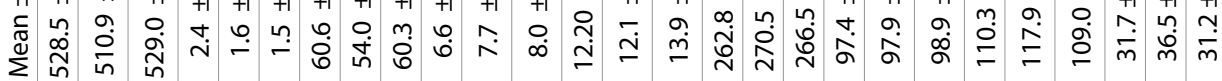

\begin{tabular}{|c|c|c|c|c|c|c|c|}
\hline 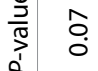 & : & $\stackrel{0}{0}$ & $\underset{0}{\Delta}$ & $\bar{o}$ & 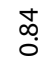 & fo & $\stackrel{8}{0}$ \\
\hline
\end{tabular}

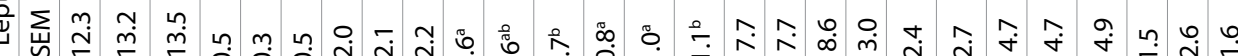

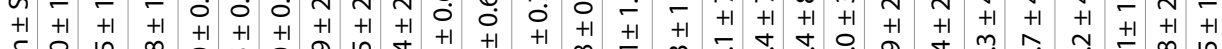

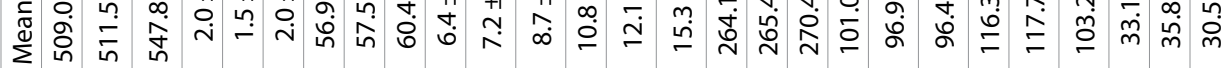

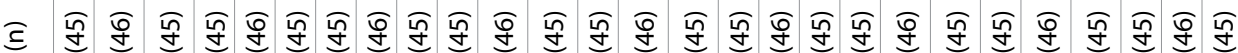

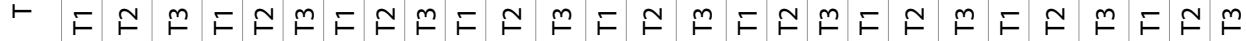




\begin{tabular}{|c|c|c|c|c|c|c|c|c|c|c|c|c|c|c|c|c|c|}
\hline \multicolumn{2}{|l|}{$\stackrel{n}{\frac{n}{0}}$} & \multicolumn{2}{|c|}{ ָ̃ } & \multicolumn{3}{|c|}{$\stackrel{\substack{\infty \\
0}}{0}$} & \multicolumn{3}{|l|}{ O্. } & \multicolumn{2}{|l|}{$\stackrel{m}{0}$} & \multicolumn{2}{|l|}{$\bar{\sigma}$} & \multicolumn{2}{|l|}{$\stackrel{t}{\Delta}$} & & \\
\hline 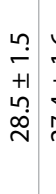 & 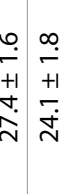 & $\begin{array}{l}\hat{r} \\
++1 \\
i n \\
\tilde{n}\end{array}$ & 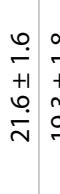 & 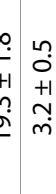 & $\begin{array}{l}+ \\
\dot{0} \\
+1 \\
+1 \\
\dot{\sigma} \\
\dot{m}\end{array}$ & $\begin{array}{l}\dot{0} \\
\dot{0} \\
+1 \\
\dot{m}\end{array}$ & 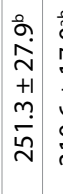 & 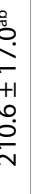 & 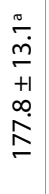 & \begin{tabular}{l|l}
0 & $\frac{0}{0}$ \\
0 & 0 \\
0 & 0 \\
0 & 0 \\
+1 & +1 \\
\hdashline & 0 \\
0 & 0
\end{tabular} & $\begin{array}{l}0 \\
\vdots \\
0 \\
+1 \\
0 \\
0\end{array}$ & 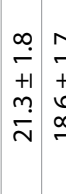 & 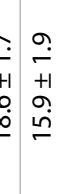 & $\begin{array}{l}0 \\
\stackrel{2}{0} \\
+1 \\
+1 \\
\\
=\end{array}$ & $\begin{array}{l}\stackrel{0}{0} \\
0 \\
+1 \\
+1 \\
+ \\
\dot{0}\end{array}$ & 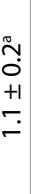 & \\
\hline \multicolumn{2}{|l|}{$\stackrel{n}{0} 0$} & \multicolumn{3}{|l|}{ 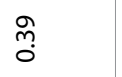 } & \multicolumn{2}{|c|}{ 令 } & \multicolumn{3}{|l|}{ Õ. } & \multicolumn{2}{|l|}{ ஸ્ } & \multicolumn{2}{|l|}{ స̃ } & \multicolumn{2}{|l|}{$\stackrel{m}{\circ}$} & & \\
\hline 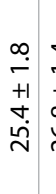 & 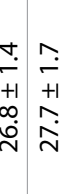 & $\begin{array}{l}\infty \\
\stackrel{\infty}{+1} \\
o \\
o \\
\sigma\end{array}$ & 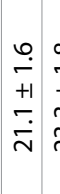 & 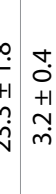 & $\begin{array}{l}0 \\
0 \\
+1 \\
10 \\
m \\
m\end{array}$ & $\begin{array}{l}n \\
0 \\
0 \\
+1 \\
0 \\
\dot{m}\end{array}$ & 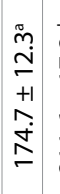 & 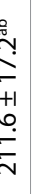 & 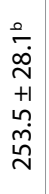 & $\begin{array}{ll}0 & 0 \\
0 & 0 \\
+1 \\
1 & +1 \\
0 & 0 \\
0\end{array}$ & 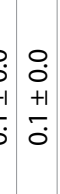 & $\begin{array}{ll}9 & 6 \\
\vdots & 7 \\
+1 & 7 \\
0 & 0 \\
0 & \alpha\end{array}$ & 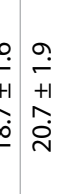 & $\begin{array}{l}\tilde{c} \\
+ \\
+1 \\
\stackrel{1}{-}\end{array}$ & & 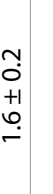 & \\
\hline \multicolumn{2}{|l|}{$\underset{\sim}{\widetilde{N}}$} & \multicolumn{2}{|c|}{$\stackrel{\circ}{\circ}$} & \multicolumn{3}{|c|}{ ڤิ } & \multicolumn{3}{|c|}{$\bar{o}_{0}$} & \multicolumn{2}{|l|}{$\stackrel{m}{0}$} & \multicolumn{2}{|l|}{$\stackrel{0}{\circ}$} & \multicolumn{2}{|l|}{$\frac{\nabla}{\circ}$} & & \\
\hline 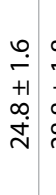 & 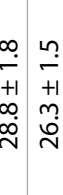 & 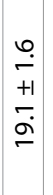 & 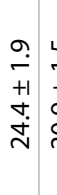 & 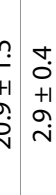 & $\begin{array}{l}n \\
0 \\
+ \\
+1 \\
n \\
n\end{array}$ & $\begin{array}{l}\dot{t} \\
0 \\
+1 \\
\stackrel{n}{m} \\
m\end{array}$ & 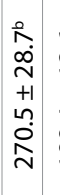 & 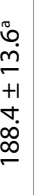 & 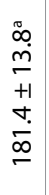 & $\begin{array}{lll} & 0 \\
0 & 0 \\
0 & 0 \\
+1 & +1 \\
& + \\
0 & 0\end{array}$ & 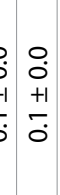 & 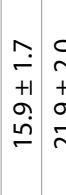 & 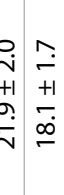 & $\begin{array}{l}+ \\
\vdots \\
0 \\
+1 \\
\stackrel{1}{1} \\
\end{array}$ & 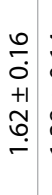 & 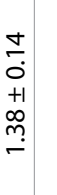 & 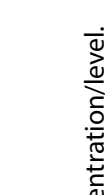 \\
\hline \multicolumn{2}{|l|}{$\stackrel{\substack{\infty \\
0}}{0}$} & \multicolumn{2}{|c|}{ 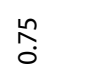 } & \multicolumn{3}{|c|}{ ণั } & \multicolumn{3}{|l|}{$i_{i}$} & \multicolumn{2}{|l|}{ ర్ర } & \multicolumn{2}{|l|}{$\stackrel{?}{\stackrel{0}{0}}$} & \multicolumn{2}{|l|}{ 苍 } & & \\
\hline 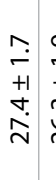 & 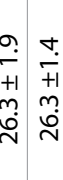 & 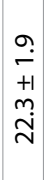 & 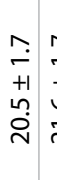 & 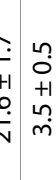 & $\begin{array}{l}+ \\
0 \\
+1 \\
+ \\
\dot{m}\end{array}$ & $\begin{array}{l}m \\
0 \\
+1 \\
\infty \\
i \\
i\end{array}$ & $\begin{array}{l}m \\
\dot{I} \\
+1 \\
+1 \\
\infty \\
\dot{N} \\
\dot{N}\end{array}$ & 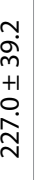 & 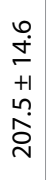 & $\begin{array}{cc}0 & 0 \\
\dot{+} & 0 \\
+1 & +1 \\
- & +1 \\
0 & 0\end{array}$ & 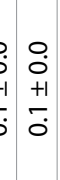 & $\begin{array}{ll}9 & 9 \\
& 7 \\
+1 & + \\
0 & 1 \\
0 & 1 \\
7 & 7\end{array}$ & 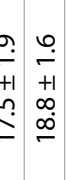 & $\begin{array}{l}r \\
0 \\
+1 \\
n \\
n\end{array}$ & $\begin{array}{l}\tilde{c} \\
0 \\
+1 \\
\\
-\end{array}$ & $\begin{array}{l}-\overline{0} \\
+1 \\
+1 \\
+ \\
\\
\end{array}$ & 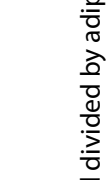 \\
\hline \multicolumn{2}{|l|}{$\stackrel{\text { to }}{\circ}$} & \multicolumn{2}{|c|}{$\underset{丶}{\stackrel{\Xi}{0}}$} & \multicolumn{3}{|c|}{ รี } & \multicolumn{3}{|l|}{$\stackrel{\tilde{0}}{0}$} & \multicolumn{2}{|l|}{ ?̊? } & \multicolumn{2}{|l|}{$\div$} & $\stackrel{\infty}{0}$ & & & $\underline{\underline{a}}$ \\
\hline 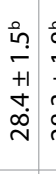 & 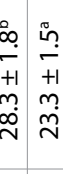 & 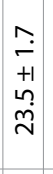 & 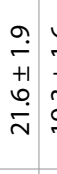 & 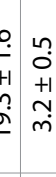 & $\begin{array}{l}m \\
0 \\
+1 \\
0 \\
0 \\
m\end{array}$ & $\begin{array}{l}+ \\
0 \\
+1 \\
o \\
i \\
i\end{array}$ & 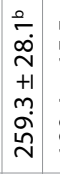 & 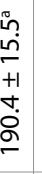 & 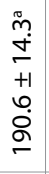 & $\begin{array}{ll}\bar{\delta} & \overline{0} \\
0 & 0 \\
+1 & +1 \\
-1 & \overline{0} \\
& 0\end{array}$ & 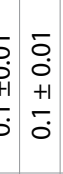 & 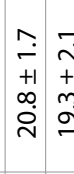 & 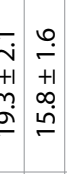 & $\begin{array}{l}- \\
+ \\
+1 \\
0 \\
-\end{array}$ & $\begin{array}{l}\text { ?̦ } \\
0 \\
+1 \\
\\
\end{array}$ & 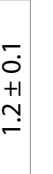 & 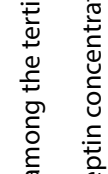 \\
\hline $\begin{array}{l}\sqrt{n} \\
F \\
F\end{array}$ & 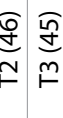 & 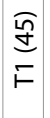 & 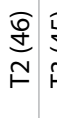 & $\begin{array}{l}\frac{\widehat{a}}{\underline{y}} \\
\underline{F}\end{array}$ & 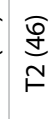 & $\begin{array}{l}\frac{\tilde{g}}{\mathrm{f}} \\
\tilde{m}\end{array}$ & $\begin{array}{l}\frac{\sqrt{g}}{8} \\
F\end{array}$ & 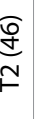 & 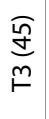 & $\begin{array}{ll}\frac{G}{d} \\
F \\
F\end{array}$ & 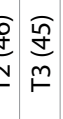 & $\begin{array}{l}\frac{\pi}{y} \\
F \\
F \\
\stackrel{0}{f}\end{array}$ & 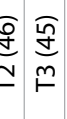 & 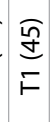 & 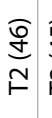 & $\stackrel{\substack{n \\
m}}{r}$ & 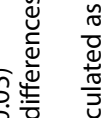 \\
\hline 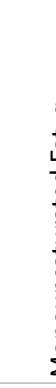 & 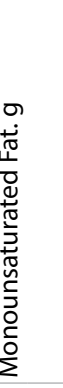 & & 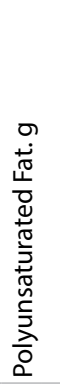 & & 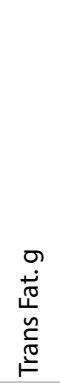 & & 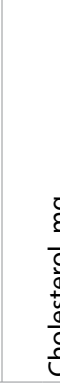 & 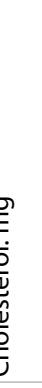 & & 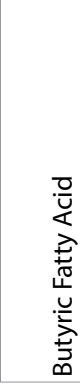 & 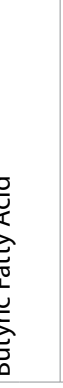 & 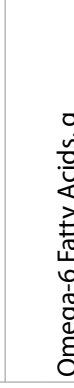 & 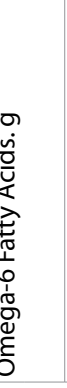 & & 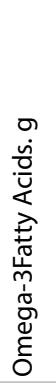 & & 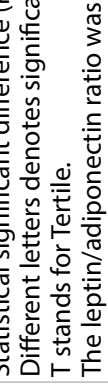 \\
\hline
\end{tabular}




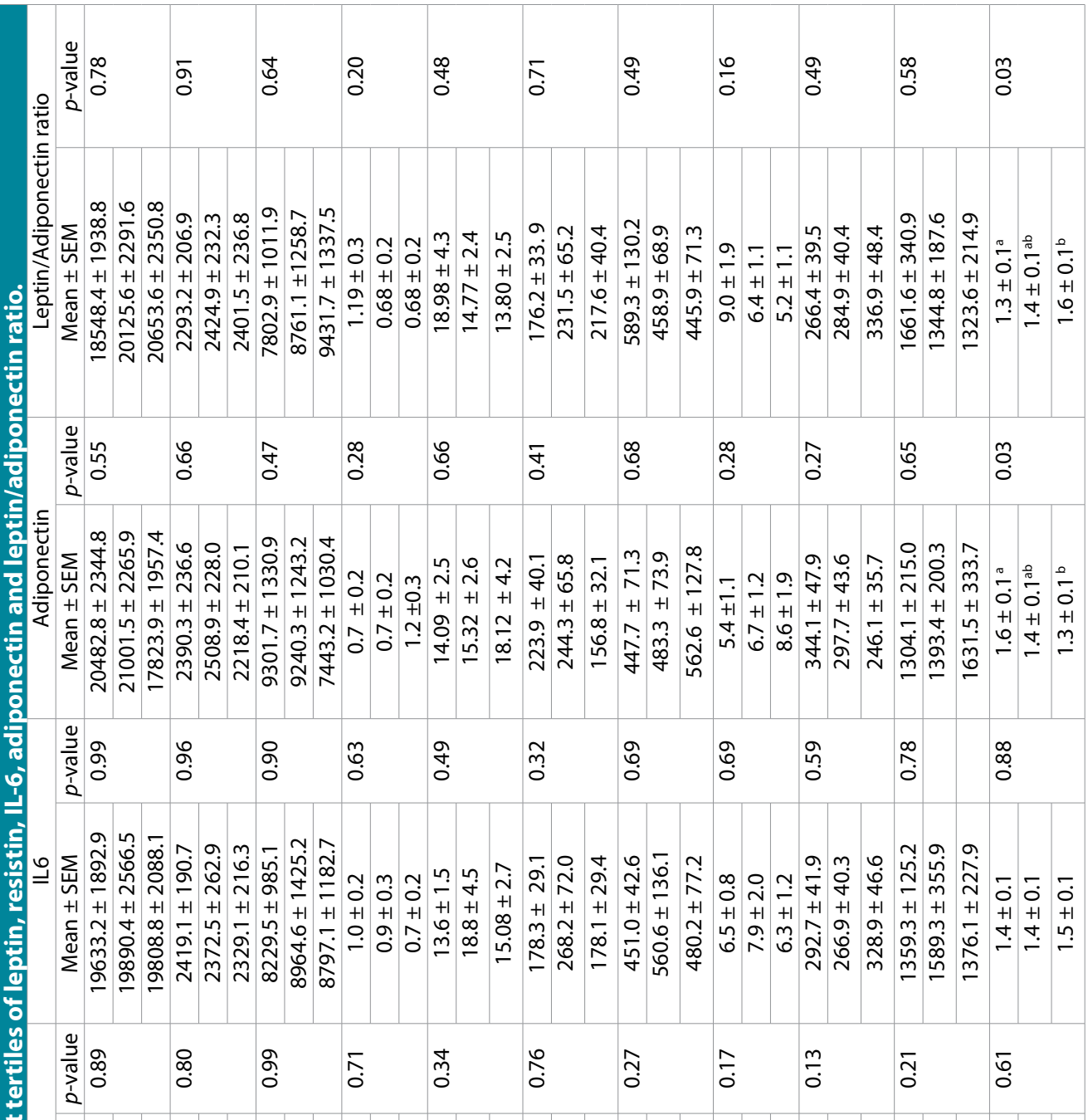

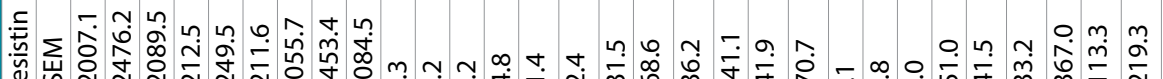

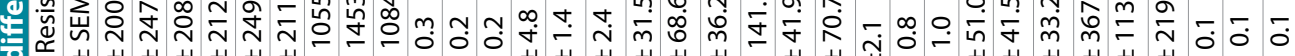

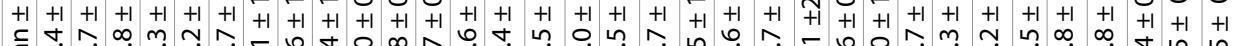

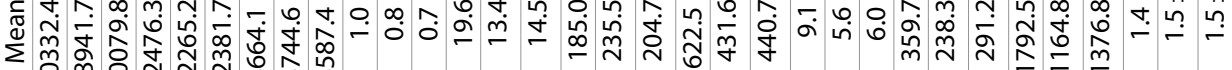

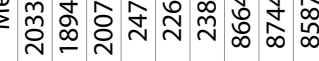

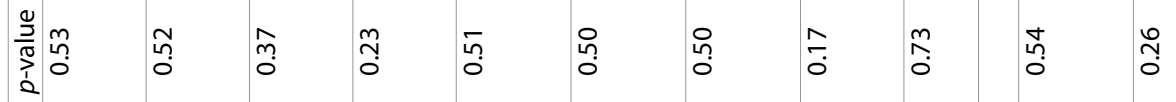

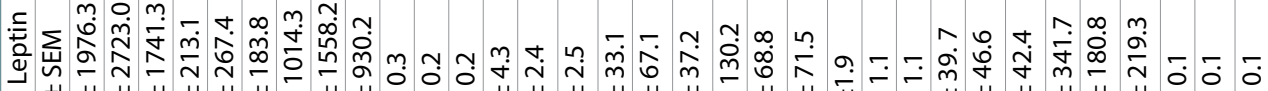

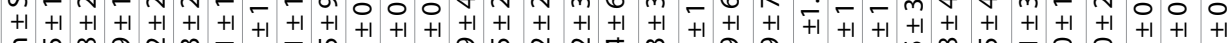

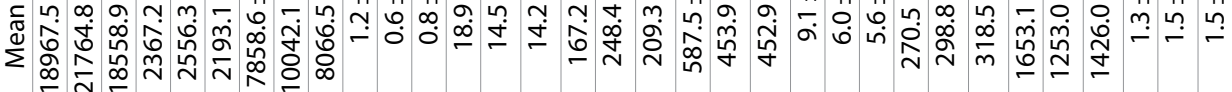

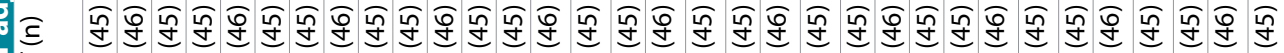

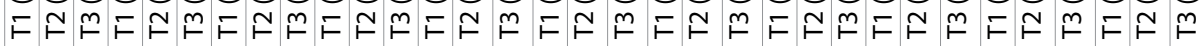

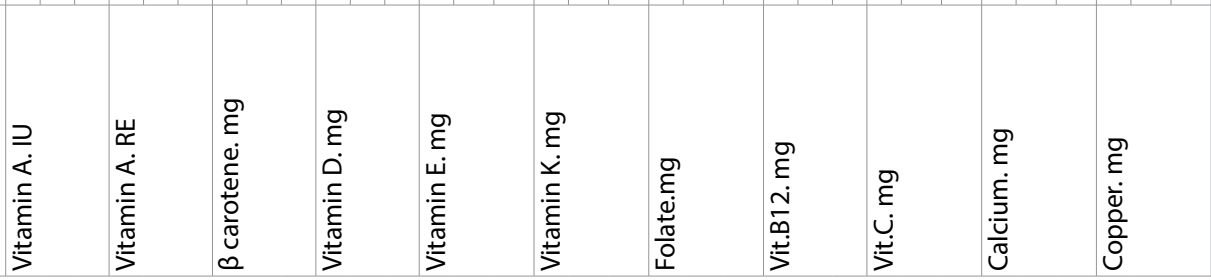




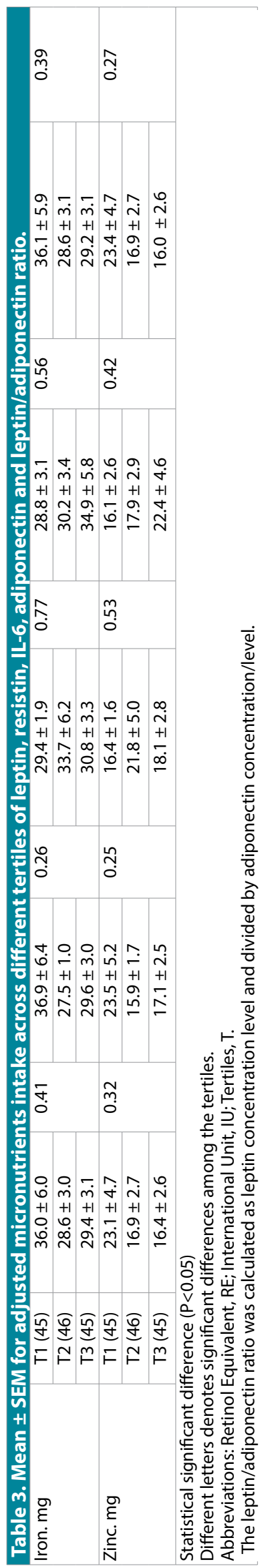




\section{Discussion}

Dietary components may modulate the risk of diseases by altering the levels of adipokines secretion and/or sensitivity [33,44,45]. In this study of Jordanian adults, we highlighted a few associations between dietary consumption of macro- and micro-nutrients and serum levels of leptin, adiponectin, IL-6, resistin, as well as leptin/adiponectin ratio.

The consumption of carbohydrate plays an integral role in the regulation of leptin level and adiponectin due to the concurrent changes in glucose homeostasis and insulin secretion $[44,46]$. Adiponectin/leptin ratio may provide a reliable measure of insulin sensitivity [47]. A high carbohydrate diet promotes glucose intolerance and insulin secretion as well as reduces insulin sensitivity, therefore, increases leptin, an insulinresistance surrogate, and reduces adiponectin, an insulin sensitivity surrogate $[46,48,49]$. A higher consumption of carbohydrate has been found to augment leptin level [44], which is in agreement with the positive association between serum leptin level and energy percent from carbohydrates that has been detected in this study. In contrast, circulating adiponectin concentration was negatively associated with glycemic load and glycemic index [20]. Also, an increased consumption of total carbohydrate has been found to be associated with a reduced level of circulating adiponectin $[18,20,50]$, which supports our finding of a higher carbohydrate consumption (percent of energy from carbohydrate and amount of carbohydrate) among the lowest tertile of serum adiponectin.

In contrast to other studies, this study found positive associations between leptin and leptin/adiponectin levels and fiber consumption (soluble and insoluble) as well as a negative association between insoluble fiber and adiponectin. Additionally, the middle tertile of serum resistin was found to be associated with the lowest fiber consumption compared to the lowest and highest tertiles. An increased fiber consumption is associated with an enhanced dietary carbohydrate quality, and consequently with better glycemic control and insulin sensitivity [50]. Additionally, fiber consumption has been previously reported to be associated with an increased level of adiponectin and a decreased level of leptin [44,50-53]. Elevated fiber intake may play a protective role against systemic inflammation [52], but the evidence regarding the association between fiber consumption and resistin is still controversial.
One dietary fiber-derivative, propionic acid, was found to inhibit the expression level of resistin, a component of inflammation and insulin resistance [54]. The consumption of a healthy dietary pattern was associated with a significant reduction in resistin level in a large sample of apparently healthy women and the researchers suggested that the effect was mediated, partly, by cereal fiber consumption [35]. However, Parikh et al. (2012) reported a lack of association between resistin level and fiber consumption [52]. To the best of our knowledge, higher fiber consumption generally enhances the sensitivity of insulin and leptin, reduces the circulating concentration of leptin, and increases the circulating adiponectin level. Therefore, it is hard to give a convincing explanation for the detected associations between fiber consumption and levels of adipokines, these associations would highlight the diet complexity and the importance of overall diet quality, where a high fiber intake does not necessarily mean healthier choices or better diet quality.

The consumption of a high amount of dietary fat elevates the circulating leptin level and eventually reduces the cellular responsiveness to leptin $[27,55]$. Unlike saturated fatty acid, monounsaturated and polyunsaturated fatty acids were found to reduce the circulating concentrations of leptin [44]. Results from the present study identified negative associations between serum leptin level and the percent energy from fat as well as the amount of monounsaturated fatty acid consumed. The association between fat consumption and leptin concentration is provocative; with results indicating a positive [56,57], negative [58], and no association [59]. This might be due to the variation in participants characteristics, study design, dietary fat quality, or overall diet quality. While most of the available evidence assessed the effect of polyunsaturated and saturated fatty acid on circulating leptin levels [60-62], little is known about the effect of monounsaturated fatty acid. However, the Mediterranean diet, monounsaturated fat-rich diet, was found to reduce leptin level. Dietary monounsaturated fat was found to significantly reduce serum leptin compared to saturated fatty acid and carbohydrate [63], which might be, partly, explained by the beneficial effect of the consumption of monounsaturated fatty acid on insulin sensitivity [63,64]. Further research is warranted to evaluate the effect of monounsaturated fatty acid consumption on leptin levels. 
In this study, higher cholesterol consumption was found to be associated with a higher level of adiponectin and lower levels of leptin, IL6, and leptin/adiponectin ratio. Cholesterol synthesis and absorption have been found to be associated with serum levels of several adipokines including leptin and adiponectin [65]. The association between serum levels of adipokines and cholesterol metabolism challenges the explanation of the association between cholesterol consumption and serum levels of these adipokines. Also, the cholesterol consumption of the participant in this study was within the normal range $(<300 \mathrm{mg} / \mathrm{dL})$ which further challenges the interpretation of these association.

Regarding the detected association between butyric acid and leptin/adiponectin ratio, it was not possible to find any association between dietary butyric acid and adiponectin or leptin during the present study. However, shortchain fatty acids have been found to improve glycemic control, modulate nutrient trafficking and oxidation, modulate the expression level of several proteins/enzymes, that regulate energy balance including leptin, and may positively affect body weight control [54,66,67]. Given the involvement of leptin and adiponectin in glucose and energy metabolism, the negative association between butyric acid consumption and leptin/adiponectin ratio that has been detected in the current study, may reflect the beneficial metabolic effect of butyric acid. More studies are needed to support this association.

Participants in the highest tertile of leptin/ adiponectin ratio had a significantly lower intake of omega-3 fatty acid compared to those in the lowest tertile. Omega-3 fatty acid was previously reported to modulate the levels of adiponectin, leptin and adiponectin/leptin ratio. Serum adiponectin levels significantly increased following 8 -week of $3 \mathrm{~g} / \mathrm{d}$ of omega-3 fatty acid supplementation in healthy young female [29]. Also, an increased dietary consumption of fish was found to be associated with a reduced level of leptin [28]. Mostowik et al. (2013) found that daily supplementation of $1 \mathrm{~g} / \mathrm{d}$ of omega-3 fatty acid for 30 days significantly increased adiponectin level and adiponectin/leptin ratio as well as resulted in a significant reduction in leptin level [68]. The effect of omega-3 fatty acid on adiponectin and leptin levels is suggested to be a concomitant to its modulatory effects on cytokine and proinflammatory mediators [21].

Data concerning the association between adipokine levels and copper consumption is scarce, however, copper and adiponectin are involved in several similar metabolic processes including oxidative defense system and glycemic control, in which the levels of adiponectin and copper were negatively, associated with the risk factors of abnormal health conditions such as diabetes, cardiovascular disease, and oxidative stress [69-71]. Additionally, copper has been suggested as a possible mediator of leptin levels regulation [72]. A positive association between serum level of leptin and serum level of copper, a surrogate marker of its consumption, has been reported in healthy adult as well as in female athletes [72,73]. Therefore, findings of this study might reflect a possible role of copper in the regulation of circulating level of adiponectin. Further research is warranted to test the possible modulatory effect of copper consumption on serum adiponectin levels.

One of the strong points of this study is the use of a validated Arabic FFQ that was modified to reflect the food consumption pattern in Arab countries, especially Jordan. Furthermore, the strength of this study can be attributed to the use of food models and measurements tools to help the participants to estimate portion sizes. The main limitation of this study, however, is the one year dietary recall period, which may be affected by behavioral and dietary changes, memory and bias. Cross-sectional study design cannot assess the cause and effect relationship between nutrients and adipokines level. In addition, our sample size is small due to the limited financial support for the biochemical analyses. Therefore, we recommend conducting additional studies to detect the effect not just the association between nutrients intake and the level of serum adipokines. Additionally, another study on a large-scale is warranted to generalize these findings.

In conclusion, the results of the present study highlighted that the consumption of some nutrients (carbohydrates, fibers, fats, monounsaturated fats, cholesterol and copper) may ameliorate the circulating adipokines in different aspects.

\section{Acknowledgments}

The authors would like to thank the Royal Medical Services for their great help and support and for allowing us to conduct this study in their nutrition clinic. 


\section{Conflict of interests}

The authors declare that they have no conflict of interests.

\section{Authority \\ RFT NSH and AMA participated in}

conception and design of the study. NAN, LMA and SSA entered data and performed biochemical analysis and statistical analysis. RFT, NSH, BAB, SH, and AMA interpreted the study results and drafted the manuscript. 


\section{REFERENCES}

1. Hausman DB, DiGirolamo M, Bartness TJ, Hausman GJ, Martin RJ. The biology of white adipocyte proliferation. Obes. Rev. 2(4), 239-254 (2001).

2. Kershaw E, Flier JS. Adipose tissue as an endocrine organ.J. Clin. Endocrinol. Metab. 89(6), 2548-2556 (2004).

3. Galic S, Oakhill JS, Steinberg GR. Adipose tissue as an endocrine organ. Mol. Cell Endocrinol. 316(2), 129-139 (2010).

4. Gualillo O, Gonzalez-Juanatey JR, Lago F. The emerging role of adipokines as mediators of cardiovascular function: physiologic and clinical perspectives. Trends Cardiovasc. Med. 17(8), 275-283 (2007).

5. Bacha F, Saad R, Gungor N, Arslanian SA. Adiponectin in youth: relationship to visceral adiposity, insulin sensitivity, and beta-cell function. Diabetes Care 27(2), 547-552 (2004).

6. Hivert MF, Sullivan LM, Fox CS, et al. Associations of adiponectin, resistin, and tumor necrosis factor-alpha with insulin resistance. J. Clin. Endocrinol. Metab. 93(8), 3165-3172 (2008).

7. Shibata R, Sato K, Pimentel DR, et al. Adiponectin protects against myocardial ischemia-reperfusion injury through AMPK- and COX-2dependent mechanisms. Nat. Med. 11(10), 1096-1103 (2005).

8. Cheng KK, Lam KS, Wang Y, et al. Adiponectin-induced endothelial nitric oxide synthase activation and nitric oxide production are mediated by APPL1 in endothelial cells. Diabetes 56(5), 1387-1394 (2007).

9. Zhang Y, Proenca R, Maffei M, et al. Positional cloning of the mouse obese gene and its human homologue. Nature 372(6505), 425-432 (1994).

10. Friedman JM, Halaas JL. Leptin and the regulation of body weight in mammals. Nature 395(6704), 763770 (1998).

11. Yannakoulia M, Yiannakouris N, Bleuher $S$, et al. Body fat mass and macronutrient intake in relation to circulating soluble leptin receptor, free leptin index, adiponectin, and resistin concentrations in healthy humans. J. Clin. Endocr .Metab. 88(4), 17301736 (2003).

12. Tschritter O, Fritsche A, Thamer C, et al. Plasma adiponectin concentrations predict insulin sensitivity of both glucose and lipid metabolism. Diabetes 52(2), 239-243 (2003).

13. Rotter V, Nagaev I, Smith U. Interleukin-6 (IL-6) induces insulin resistance in 3T3-L1 adipocytes and is, like IL-8 and tumor necrosis factoralpha, overexpressed in human fat cells from insulin-resistant subjects. J. Biol. Chem. 278(46), 45777-45784 (2003).

14. Rajala MW, Qi Y, Patel HR, et al. Regulation of resistin expression and circulating levels in obesity, diabetes, and fasting. Diabetes 53(7), 16711679 (2004).

15. Meigs JB, Dupuis J, Liu C, et al. PAI1 Gene $4 \mathrm{G} / 5 \mathrm{G}$ polymorphism and risk of type 2 diabetes in a population based sample. Obesity 14(5), 753-758 (2006).

16. Matsuzawa Y. Therapy insight: adipocytokines in metabolic syndrome and related cardiovascular disease. Nat. Clin. Pract. Cardiovasc. Med. 3(1), 3542 (2006).

17. Despres JP Lemieux I. Abdominal obesity and metabolic syndrome. Nature 444(7121), 881-887 (2006).

18. Cassidy A, Skidmore P, Rimm EB, et al. Plasma adiponectin concentrations are associated with body composition and plant-based dietary factors in female twins. J. Nutr. 139(2), 353-358 (2009).

19. Mar BM, Maffeis C, Llompart I, Pons A, Tur JA. Dietary factors associated with subclinical inflammation among girls. Eur. J. Clin. Nutr. 67(12), 12641270 (2013).

20. Pischon T, Girman CJ, Rifai N, Hotamisligil GS, Rimm EB. Association between dietary factors and plasma adiponectin concentrations in men. Am. J. Clin. Nutr. 81(4), 780786 (2005).

21. Gray B, Steyn F, Davies PS, Vitetta L. Omega-3 fatty acids: a review of the effects on adiponectin and leptin and potential implications for obesity management. Eur. J. Clin. Nutr. 67(12), 1234-1242 (2013).

22. Ferranti EP, Reilly BB, Dunbar CM. Dietary omega-3 fatty acids and fiber intake increase adiponectin levels in African American adults with metabolic syndrome. Circulation 132(3), (2015).

23. Weigle DS, Breen PA, Matthys CC, et al. A high-protein diet induces sustained reductions in appetite, ad libitum caloric intake, and body weight despite compensatory changes in diurnal plasma leptin and ghrelin concentrations. Am. J. Clin. Nutr. 82(1), 41-48 (2005).

24. Vahamiko S, Isolauri E, Pesonen U, et al. Dietary sucrose intake is related to serum leptin concentration in overweight pregnant women. Eur. J. Nutr. 49(2), 83-90 (2009).

25. Kuroda M, Ohta M, Okufuji T, et al. Frequency of soup intake and amount of dietary fiber intake are inversely associated with plasma leptin concentrations in Japanese adults. Appetite 54(3), 538-543 (2010).

26. Yeon JY, Kim HS, Sung MK. Diets rich in fruits and vegetables suppress blood biomarkers of metabolic stress in overweight women. Prev. Med. 54(1), S109-S115 (2012).

27. Yannakoulia M, Yiannakouris N, Melistas L, et al. A dietary pattern characterized by high consumption of whole-grain cereals and low-fat dairy products and low consumption of refined cereals is positively associated with plasma adiponectin levels in healthy women. Metabolism 57(6), 824-830 (2008).

28. Winnicki M, Somers VK, Accurso V, et al. Fish-rich diet, leptin, and body mass. Circulation 106(3), 289-291 (2002). 
29. Kondo K, Morino K, Nishio Y, et al. Effects of a fish-based diet on the serum adiponectin concentration in young, non-obese, healthy Japanese subjects. J. Atheroscler. Thromb. 7(6), 628-637 (2010).

30. Zhang Z, Lanza E, Ross AC, et al. A high-legume low glycemic index diet reduces fasting plasma leptin in middle-aged insulin-resistant and -sensitive men. Eur. J. Clin. Nutr. 65, 415-418 (2011).

31. Al-Delaimy WK, Natarajan L, Rock CL, et al. Insulin-like growth factor I, insulin-like growth factor I binding protein 1 , insulin, glucose, and leptin serum levels are not influenced by a reduced-fat, highfiber diet intervention. Cancer Epidemiol. Biomarkers Prev. 15(6), 1238-1239 (2006).

32. Ganji V, Kafai MR, McCarthy E. Serum leptin concentrations are not related to dietary patterns but are related to sex, age, body mass index, serum triacylglycerol, serum insulin, and plasma glucose in the US population. Nutr. Metab. 6, 3 (2009).

33. Kashino I, Nanri A, Kurotani K, et al. Association of dietary patterns with serum adipokines among Japanese: a cross-sectional study. Nutr. J. 14, 58 (2015).

34. Al-Daghri NM, Al-Othman A, Alkharfy KM, et al. Assessment of selected nutrients intake and adipocytokines profile among Saudi children and adults. Endocrine J. 59(12), 1057-1063 (2012).

35. Kim J, Ahn J. Effect of Zinc Supplementation on Inflammatory Markers and Adipokines in Young Obese Women. Biological Trace Element Res. 157(2), 101-106 (2014).

36. Fargnoli JL, Fung TT, Olenczuk $\mathrm{DM}$, et al. Adherence to healthy eating patterns is associated with higher circulating total and highmolecular-weight adiponectin and lower resistin concentrations in women from the Nurses' Health
Study. Am. J. Clin. Nutr. 88(5), 1213-1224 (2008).

37. Fragopoulou E, Panagiotakos DB, Pitsavos C, et al. The association between adherence to the Mediterranean diet and adiponectin levels among healthy adults: the ATTICA study. J. Nutr. Biochem. 21(4), 285-289 (2010).

38. de León AC, González DA, Hernández AG, et al. Relationships between serum resistin and fat intake, serum lipid concentrations and adiposity in the general population. J. Atheroscler. Thromb. 21(5), 454-462 (2014).

39. Aeberli I, Gerber PA, Hochuli M, et al. Low to moderate sugarsweetened beverage consumption impairs glucose and lipid metabolism and promotes inflammation in healthy young men: a randomized controlled trial. Am. J. Clin. Nutr. 94(2), 479485 (2011).

40. Pollock NK, Bundy V, Kanto W, Davis CL, Bernard PJ, Zhu H, et al. Greater fructose consumption is associated with cardiometabolic risk markers and visceral adiposity in adolescents. J. Nutr. 142(2), 251257 (2012).

41. Tayyem RF, Abu-Mweis SS, Bawadi HA, Agraib L, BaniHani K. Validation of a Food Frequency Questionnaire to assess macronutrient and micronutrient intake among Jordanians. J. Acad. Nutr. Diet. 114, 1046-1052 (2014).

42. Pellet P, Shadarevian S. The Food Composition Tables for Use in the Middle East. 2nd, Heidelberg, 1970; AUB, Lebanon.

43. Al-Radaideh A, Tayyem R, AlFayomi K, et al. Assessment of Abdominal Fat Using High-field Magnetic Resonance Imaging and Anthropometric and Biochemical Parameters. Am. J. Med. Sci. 3526, 593-602 (2016).

44. Izadi V, Saraf-Bank S, Azadbakht L. Dietary intakes and leptin concentrations. ARYA Atheroscler.
10(5), 266-272 (2014).

45. Olivares-Garcia V, Torre-Villalvazo I, Velazquez-Villegas L, et al. Fasting and postprandial regulation of the intracellular localization of adiponectin and of adipokines secretion by dietary fat in rats. Nutr. Diabetes 5, e184 (2015).

46. Ko BJ, Park KH, Mantzoros CS. Diet patterns, adipokines, and metabolism: where are we and what is next? Metabolism 63(2), 168-177 (2014).

47. Zaletel J, Barlovic DP, Prezelj J. Adiponectin-leptin ratio: a useful estimate of insulin resistance in patients with Type 2 diabetes. J. Endocrinol. Invest. 33(8), 514-518 (2010).

48. Askari H, Tykodi G, Liu J, DagogoJack S. Fasting plasma leptin level is a surrogate measure of insulin sensitivity. J. Clin. Endocrinol. Metab. 95(8), 3836-3843 (2010).

49. Singh B, Saxena A. Surrogate markers of insulin resistance: $A$ review. World J. Diabetes 1(2), 36-47 (2010).

50. AlEssa HB, Ley SH, Rosner B, et al. High Fiber and Low Starch Intakes Are Associated with Circulating Intermediate Biomarkers of Type 2 Diabetes among Women. J. Nutr. 146(2), 306-317 (2016).

51. Izadi V, Azadbakht L. Specific dietary patterns and concentrations of adiponectin. J. Res. Med. Sci. 20(2), 178-184 (2015).

52. Parikh S, Pollock NK, Bhagatwala J, et al. Adolescent fiber consumption is associated with visceral fat and inflammatory markers. J. Clin. Endocrinol. Metab. 97(8), E14511457 (2012).

53. Qi L, Meigs JB, Liu S, et al. Dietary fibers and glycemic load, obesity, and plasma adiponectin levels in women with type 2 diabetes. Diabetes Care 29(7), 1501-1505 (2006).

54. Al-Lahham SH, Roelofsen H, Priebe $\mathrm{M}$, et al. Regulation of adipokine 
production in human adipose tissue by propionic acid. Eur. J. Clin. Invest. 40(5), 401-407 (2010).

55. Morrison CD, Huypens P, Stewart LK, Gettys TW. Implications of crosstalk between leptin and insulin signaling during the development of diet-induced obesity. Biochim. Biophys. Acta 1792(5), 409-416 (2009).

56. Chu NF, Stampfer MJ, Spiegelman $\mathrm{D}$, et al. Dietary and lifestyle factors in relation to plasma leptin concentrations among normal weight and overweight men. Int. J. Obes. Relat. Metab. Disord. 25(1), 106-114 (2001).

57. Fung TT, Rimm EB, Spiegelman D, et al. Association between dietary patterns and plasma biomarkers of obesity and cardiovascular disease risk. Am. J. Clin. Nutr. 73(1), 61-67 (2001).

58. Havel PJ, Townsend R, Chaump L, Teff K. High-fat meals reduce 24-h circulating leptin concentrations in women. Diabetes 48(2), 334-341 (1999).

59. Lovejoy JC, Windhauser MM, Rood JC, de la Bretonne JA. Effect of a controlled high-fat versus lowfat diet on insulin sensitivity and leptin levels in African-American and Caucasian women. Metabolism 47(12), 1520-1524 (1998).

60. Reseland JE, Haugen F, Hollung $\mathrm{K}$, et al. Reduction of leptin gene expression by dietary polyunsaturated fatty acids. J. Lipid Res. 42(5), 743-750 (2001).

61. Wang H, Storlien LH, Huang
XF. Effects of dietary fat types on body fatness, leptin, and ARC leptin receptor, NPY, and AgRP mRNA expression. Am. J. Physiol. Endocrinol. Metab. 282(6), E13521359 (2002).

62. Yamasaki M, Ikeda A, Oji M, et al. Modulation of body fat and serum leptin levels by dietary conjugated linoleic acid in Sprague-Dawley rats fed various fat-level diets. Nutrition 19(1), 30-35 (2003).

63. Paniagua JA, Gallego de la Sacristana A, Romero I, et al. Monounsaturated fat-rich diet prevents central body fat distribution and decreases postprandial adiponectin expression induced by a carbohydrate-rich diet in insulin-resistant subjects. Diabetes Care 30(7), 1717-1723 (2007).

64. Vessby B, Uusitupa M, Hermansen $\mathrm{K}$, et al. Substituting dietary saturated for monounsaturated fat impairs insulin sensitivity in healthy men and women: The KANWU Study. Diabetologia. 44(3), 312-319 (2001).

65. Hallikainen M, Kolehmainen M, Schwab U, Laaksonen DE, Niskanen L, Rauramaa R, et al. Serum adipokines are associated with cholesterol metabolism in the metabolic syndrome. Clin. Chim. Acta. 383(1-2), 126-132 (2007).

66. Besten G, van Eunen K, Groen AK, et al. The role of short-chain fatty acids in the interplay between diet, gut microbiota, and host energy metabolism. J. Lipid Res. 54(9), 2325-2340 (2013).

67. Marinangeli CP, Jones PJ. Pulse grain consumption and obesity: effects on energy expenditure, substrate oxidation, body composition, fat deposition and satiety. Br. J. Nutr. 108 Suppl 1: S46-51 (2012).

68. Mostowik M, Gajos G, Zalewski J, Nessler J, Undas A. Omega-3 polyunsaturated fatty acids increase plasma adiponectin to leptin ratio in stable coronary artery disease. Cardiovasc. Drugs Ther. 27(4), 289295 (2013).

69. Bo S, Durazzo M, Gambino R, Berutti C, Milanesio N, Caropreso A, et al. Associations of dietary and serum copper with inflammation, oxidative stress, and metabolic variables in adults. J. Nutr. 138(2), 305-310 (2008).

70. Chan KH, Lam KS, Cheng OY, et al. Adiponectin is protective against oxidative stress induced cytotoxicity in amyloid-beta neurotoxicity. PLoS One 7(12), e52354 (2012).

71. Uriu-Adams JY, Keen CL. Copper, oxidative stress, and human health. Mol. Aspects Med. 26(4-5), 268298 (2005).

72. Casimiro-Lopes G, de OliveiraJunior AV, Portella ES, Lisboa PC, et al. Plasma leptin, plasma zinc, and plasma copper are associated in elite female and male judo athletes. Biol. Trace Elem. Res. 127(2), 109-115 (2009).

73. Olusi S, Al-Awadhi A, Abiaka C, Abraham M, George S. Serum copper levels and not zinc are positively associated with serum leptin concentrations in the healthy adult population. Biol. Trace Elem. Res. 91(2), 137-144 (2003). 